\title{
Correlation analysis of circulating myeloma cells with tumor load, angiogenesis and disease progression of multiple myeloma and clinical significance
}

\section{Ran Tan ( 1019957212@qq.com )}

Zhengzhou University First Affiliated Hospital

Yuke Liu

Zhengzhou University First Affiliated Hospital

Qiyao Pu

Zhengzhou University First Affiliated Hospital

Jiwei Li

Zhengzhou University First Affiliated Hospital

\section{Xueyan Cao}

Zhengzhou University First Affiliated Hospital

\section{Baohong Yue}

Zhengzhou University First Affiliated Hospital https://orcid.org/0000-0001-6911-0177

\section{Research Article}

Keywords: Multiple myeloma, Circulating myeloma cells, Marrow myeloma cells, Flow cytometry

Posted Date: February 2nd, 2022

DOl: https://doi.org/10.21203/rs.3.rs-1260412/v1

License: (c) (i) This work is licensed under a Creative Commons Attribution 4.0 International License.

Read Full License 


\section{Abstract}

Objective to investigate the correlation between the phenotype and number of circulating myeloma cells (CMCs) and the load of marrow myeloma cells (MMCs) in patients with multiple myeloma (MM) and the relationship between CMCs and MM prognostic indicators, and to evaluate the feasibility of CMCs reflecting the load of myeloma cells, angiogenesis and disease progression. 109 confirmed MM patients were selected and divided into newly diagnosed and relapse (ND/RA) group ( $n=53)$, partial remission (PR) group $(n=30)$ and complete remission (CR) group $(n=23)$ according to diagnosis and efficacy criteria. Thirty anemia patients with non-plasma cell related diseases in our hospital were selected as control group. Flow cytometry was used to detect the proportion and phenotype of CMCs and MMCs in each group. The prognostic indicators of MM patients were analyzed, including hemoglobin $(\mathrm{Hb})$, plasma albumin (Alb), beta2-microglobulin ( $\beta 2-\mathrm{MG}$ ), serum creatinine (sCrea), serum calcium (Ca), lactate dehydrogenase $(\mathrm{LDH})$, bone destruction, renal impairment, Durie-Salmon stage and International Staging System stage. There was a positive correlation between CMCs and MMCs in ND/RA and PR groups $(P<0.05)$; The proportions of MMCs and CMCs in ND/RA group were significantly higher than those in PR, $\mathrm{CR}$ and control groups $(\mathrm{P}<0.05)$. The expression rate of cytoplasmic vascular endothelial growth factor(cyVEGF) in CMCs was significantly higher than that in circulating plasma cells. Therefore, we concluded that CMCs can reflect tumor load, angiogenesis and disease progression in MM patients. Detection of CMCs improves patient compliance compared to MMCs.

\section{Introduction}

Multiple myeloma (MM) is a malignant hematologic disease characterized by abnormal proliferation of neoplastic plasma cells in bone marrow, often accompanied by multiple lytic lesions, hypercalcemia, anemia and renal damage. It occurs in middle-aged and elderly people and is still incurable [1]. Factors associated with the prognosis of patients with MM are diverse and complex. A study shows that tumor cells in bone marrow is an important prognostic factor in patients with $\mathrm{MM}$, but the examination of marrow myeloma cells needs to extract the patient's bone marrow. Extracting marrow is complicated and has shortcomings, such as bone marrow liquid is easy to be diluted, so the patients' compliance is greatly reduced [2]. Studies [3-9] have shown that there are a small amount of circulating myeloma cells (CMCs) in the peripheral blood of MM patients. CMCs are believed to reflect the tumor load independently $[4,5,7$, 9]. It is of great significance for MM staging [7, 9], prognosis [3, 4] and efficacy evaluation [6, 8]. If peripheral blood can be collected to detect CMCs reflecting tumor load, patient compliance will be significantly improved. The purpose of this study was to explore the phenotypic characteristics of CMCs, the correlation between CMCs and the proportion of marrow myeloma cells (MMCs), and the relationship between CMCs and prognostic indicators of MM, so as to evaluate the feasibility of CMCs reflecting tumor load and disease progression in MM patients.

\section{Materials And Methods}




\section{Patients}

Patients were selected according to the following inclusion and exclusion criteria. Inclusion criteria: All the selected patients met the diagnostic criteria of MM in the National Comprehensive Cancer Network (NCCN) [10]. All the selected patients were conscious and able to express their wishes. None of the selected patients had complicated diseases of other vital organs. Exclusion criteria: Patients with severe hemolytic disease. Patients with tumors in other parts. Patients with heart, liver, kidney and other important organ function impairment. Patients suffering from serious infectious diseases. A total of 109 patients with MM diagnosed in the First Affiliated Hospital of Zhengzhou University from October 2020 to April 2021 were selected, including 62 male patients and 47 female patients. The average age was $(60.0 \pm 8.5)$ years (41 81 years). According to Durie Salmon (DS) staging system [11], 12 cases were in $\Downarrow$

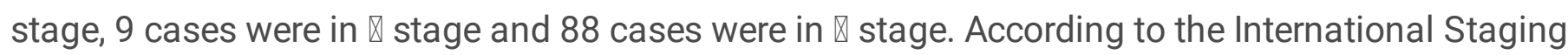
System (ISS) [12], 28 patients were in $\nabla$ stage, 28 cases were in $\nabla$ stage and 53 patients were in $\nabla$ stage. According to the guidelines for the diagnosis and treatment of multiple myeloma [1], 109 patients with MM were divided into ND/RA group $(n=56)$, PR group $(n=30)$ and CR group $(n=23)$. At the same time, 30 patients with anemia but non-plasma cells related disorders (control group) were selected including 17 males and 13 females, aged 53(33 83) years. This study was approved by the Medical Research Ethics Committee of the First Affiliated Hospital of Zhengzhou University.

\section{Reagents and instruments}

Monoclonal fluorescent antibodies, isotype control antibodies and cell permeable membrane solution (PERM) were purchased from Beeton Dickinson, USA. The instruments used were BD Facs Diva and FACS

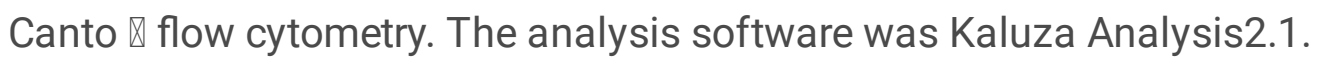

\section{Specimen pretreatment}

One day before the first chemotherapy for newly diagnosed MM patients, one day after 2 - 6 cycles of regular chemotherapy for relapse, PR and CR patients, and one day before routine clinical treatment for anemia patients in the control group, $2 \mathrm{~mL}$ of bone marrow and peripheral blood were collected respectively, treated with EDTA-K $\mathrm{K}_{2}$ anticoagulant, and were cleaned with PBS for 3 times within $24 \mathrm{~h}$. The cell count was adjusted to $(4-10) \times 10^{9} / \mathrm{L}$.

\section{Antibody combination strategies}

According to the recommendation of the European Myeloma Working Group [13], the strategy was developed for bone marrow specimens like CD 56-FITC / CD 38-PE / CD27-PerCP / CD117-PE-CY7 / CD138-APC / CD45-V450 / CD19-BV605』cKappa-FITC / cLambda-PE / CD56-PE-CY7 / CD38-APC / CD45V450 / CD19-BV605 and isotype control IgG1-FITC / IgG1-PE / CD45-PerCP / IgG1-PE-CY7 / IgG1-APC / IgG1-V450 / IgG1-BV605. According to relevant studies by Fujisawa[5] and Teresa[14], the strategy was developed for peripheric blood samples like HLA-DR-FITC / cVEGF-PE / CD27-PerCP / CD56-PE-CY7 / CD138-APC / CD19-APC-CY7 / CD45-V500 / CD38-BV605』cKappa-FITC / cLambda-PE / CD56-PE-CY7 / 
CD138-APC / CD19-APC-CY7 / CD45-V500 / CD38-BV605 and isotype control IgG1-FITC / IgG1-PE / CD45-PerCP / IgG1-PE-CY7 / IgG1-APC / IgG1-V450 / IgG1-BV605.

\section{Methods of antibody labeling}

Cell membrane antigen detection tubes: $\mathrm{V}$ ml bone marrow or peripheral blood was first added to each test tube, and RBC lysate was added at a ratio of $1: 20$. Then the tubes was incubated in the dark for 10 min. An appropriate amount of monoclonal antibody was added and mixed. The tubes were incubated in the dark at room temperature for $15 \mathrm{~min}$, and centrifuged at $500 \mathrm{~g}$ for $5 \mathrm{~min}$. $2 \mathrm{ml}$ PBS was added for washing once and then detected by flow cytometry. Intracellular antigen assay tubes $\square$ First, $\mathrm{V}$ ml bone marrow or peripheral blood was added, RBC lysate was added in the ratio of $1: 20$, and was incubated in the dark for $10 \mathrm{~min}$. An appropriate amount of monoclonal antibody was added for mixing, and the cells were incubated in the dark at room temperature for $15 \mathrm{~min}$. $100 \mathrm{ml}$ cell membrane permeable solution $\mathrm{A}$ was added for incubation in the dark for $5 \mathrm{~min}$, and centrifuged at $500 \mathrm{~g}$ for $5 \mathrm{~min}$. An appropriate amount of cKappa-FITC, cLambda-PE or cVEGF-PE and 50ml cell permeable membrane solution B were added for dark incubation for $15 \mathrm{~min}$, followed by centrifugation at $500 \mathrm{~g}$ for $5 \mathrm{~min}$, and supernatant was discarded. After washing with PBS once, flow cytometry was used to detect.

\section{Detection and analysis}

The sensitivity of FCM is $10^{-4}$ [9] even through acquiring a large number of cells such as 150,000 events $[7,15], 1,000,000$ events [16].1 million cells were collected per test tube. The MMCs (\%) results show percentages, and the CMCs (\%o) results show thousandth ratios. According to the definition standard of FCM for detecting small residual lesions in MM [1], the ratio of tumor cells detected by FCM was considered positive if greater than $1 \times 10^{-4}$, and negative if less than $1 \times 10^{-4}$.

\section{Statistical analysis}

SPSS 22.0 statistical software was used, and the proportion of MMCs and CMCs was expressed as the median (quartile) $\left[\mathrm{M}\left(\mathrm{P}_{25} \sim \mathrm{P}_{75}\right)\right.$ ]. Spearman rank correlation was used for association analysis, $\chi^{2}$ test was used for rate comparison, multivariate analysis was used for unconditional Logistic regression analysis, and Kruskal - Wallis $\mathrm{H}$ test was used for comparison of MMCs and CMCs proportions among groups. $\mathrm{P}<0.05$ was considered statistically significant. Mann-Whitney $U$ test was used for further pairto-pair comparison, and Bonferroni method was used to correct the test level.

\section{Results}

Comparison of the proportion of MMCs and CMCs and the positive detection rate of CMCs among all groups

The mean proportions of MMCs in ND/RA group, PR group, CR group and control group were 16.5794 ( \pm 19.4774$), 4.6098$ ( \pm 3.3411$), 0.0004( \pm 0.0008), 0.0002( \pm 0.0003) \%$, respectively. The mean proportions of CMCs were 3.0339 ( \pm 6.8702$), 0.0918$ ( \pm 0.1924$), 0.0001( \pm 0.0002), 0.0000( \pm 0.0002) \%$. There were 
statistically significant differences in the proportion of MMCs and CMCs among all groups (Both $\mathrm{P}$ values were less than 0.001). The proportions of MMCs and CMCs in ND/RA group were significantly higher than those in PR group, $C R$ group and control group (All $P$ values were less than 0.001 ). The positive rate of CMCs in ND/RA group (38/56, 67.9\%) was significantly higher than that in PR group, CR group and control group $(13 / 30,43.3 \%, 1 / 23,4.3 \%, 0 / 30,0 \%$ ) (All P values were less than 0.001 ) (Fig. 1).

\section{Correlation between CMCs and MMCs ratio}

The result of Person correlation analysis shows that there was a positive correlation between the proportion of CMCs and MMCs in the ND/RA group and PR group ( $r=0.694,0.692, P=0.000)$ (Fig. 2). There was no significant correlation between the proportion of CMCs and MMCs in CR group and control group $(r=0.164,0.177, P$ values $=0.454,0.350)$.

Expression of MMCs and CMCs antigens in MM patients and controls and comparison of cyVEGF expression rate between CMCs and circulating plasma cells

The proportion of MMCs and CMCs in 30 controls and 23 CR patients was low, scattered in FCM diagram, and there was no restricted or monoclonal expression of intracellular light chain, so it was non-specific. CD138 was expressed in 100\% (86 cases), CD38 was expressed in 100\% (86 cases), CD19 was expressed in $2.3 \%$ ( 2 cases), and CD 45 was expressed in 19.7\% (17 cases) of 56 ND/RA patients and 30 PR patients. Therefore, CD38+/CD138+ could be used to label plasma cells, and CD19- and/or CD45- could be used to reflect phenotypic abnormalities of plasma cells to detect CMCs (Fig. 30Fig. 4). The expression rate of cyVEGF in CMCs was significantly higher than that in circulating plasma cells with statistical significance $(P=0.0031)$ (Fig. 5).

Univariate analysis of CMCs and prognostic indicators in MM patients

According to the MM staging and typing standards in The Chinese Guidelines for the Diagnosis and treatment of multiple myeloma [1], relevant indicators were selected, as shown in Table 1. There was no significant difference in the proportion of patients with hypoalbuminemia, hypercalcemia and increased LDH between the two groups $(P>0.05)$, other indexes showed statistically significant differences between the two groups $(P<0.05)$. 
Table 1

Univariate analysis of CMCs and prognostic indicators in MM patients [Cases (\%)]

\begin{tabular}{|c|c|c|c|c|}
\hline Variable & $\mathrm{CMCs}^{+}(\mathrm{n}=52)$ & $\mathrm{CMCs}^{-}(\mathrm{n}=57)$ & $\chi^{2}$ & $\begin{array}{l}P \\
\text { value }\end{array}$ \\
\hline $\begin{array}{l}\text { Median age, } \\
\text { (years(range)) }\end{array}$ & $60(41-79)$ & $58(42-81)$ & 1.162 & 0.281 \\
\hline Sex, male & $27(51.9)$ & $35(61.4)$ & 0.996 & 0.318 \\
\hline Hemoglobin<85g/L & $21(40.4)$ & $2(3.5)$ & 22.221 & 0.000 \\
\hline Albumin<35g/L & $20(38.5)$ & $23(40.4)$ & 0.041 & 0.840 \\
\hline $\begin{array}{l}\text { Beta2- } \\
\text { microglobulin }>3.5 \mathrm{mg} / \mathrm{L}\end{array}$ & $39(75.0)$ & 10(17.5) & 36.277 & 0.000 \\
\hline Creatinine $>177 \mu \mathrm{mm} / \mathrm{L}$ & $15(28.8)$ & $3(5.3)$ & 10.970 & 0.001 \\
\hline Calcium>2.65mm/L & $1(1.9)$ & $0(0)$ & 1.106 & 0.293 \\
\hline $\mathrm{LDH}>245 \mathrm{U} / \mathrm{L}$ & 19(36.5) & 17(29.8) & 0.554 & 0.457 \\
\hline Bone destruction & $40(76.9)$ & $28(49.1)$ & 9.775 & 0.002 \\
\hline Renal inadequacy & $26(50.0)$ & 10(17.5) & 12.950 & 0.000 \\
\hline DS $\otimes$ stage & 48(92.3) & $40(70.2)$ & 8.564 & 0.003 \\
\hline ISS $₫$ stage & $33(63.5)$ & $20(35.1)$ & 8.763 & 0.003 \\
\hline
\end{tabular}

Multivariate Logistic regression analysis of CMCs and prognostic indicators in MM patients

Logistic regression analysis was performed with positive CMCs ( $1=$ yes, $0=n o$ ) as the dependent variable, moderate or above anemia, $\beta 2-\mathrm{MG}$ elevation, sCrea elevation, bone destruction, renal impairment ( $1=$ yes, $0=$ no) and DS and ISS stages ( $1=\mathbb{}$ stage, $0=I+\nabla$ stage) as independent variables. The results showed that positive CMCs was associated with moderate or above anemia and $\beta 2-M G(P<0.05)$, the regression coefficients were 1.781 and 1.812 , and the $O R$ values were 5.935 and 6.121 , respectively, indicating that moderate or above anemia and $32-\mathrm{MG}$ increase were independent risk factors for CMCs (Table 2). 
Table 2

Multivariate Logistic regression analysis of CMCs and prognostic indicators in MM patients

\begin{tabular}{|lllllll|}
\hline Variables & $\boldsymbol{\beta}$ & SE & Wald & P value & OR & 95\% Cl \\
\hline Hemoglobin<85g/L & 1.781 & 0.868 & 4.206 & 0.040 & 5.935 & $1.082 \sim 32.548$ \\
\hline Beta2-microglobulin>3.5mg/L & 1.812 & 0.667 & 7.382 & 0.007 & 6.121 & $1.657 \sim 22.617$ \\
\hline Creatinine $>177 \mu \mathrm{mm} / \mathrm{L}$ & 0.685 & 0.888 & 0.595 & 0.441 & 1.984 & $0.348 \sim 11.320$ \\
\hline Bone destruction & 0.735 & 0.529 & 1.926 & 0.165 & 2.085 & $0.739 \sim 5.883$ \\
\hline Renal inadequacy & 0.364 & 0.693 & 0.276 & 0.599 & 1.440 & $0.370 \sim 5.603$ \\
\hline DS $\otimes$ stage & 0.450 & 0.682 & 0.436 & 0.509 & 1.569 & $0.412 \sim 5.968$ \\
\hline ISS $\otimes$ stage & -0.630 & 0.652 & 0.934 & 0.334 & 0.533 & $0.148 \sim 1.911$ \\
\hline constant & -1.947 & 0.639 & 9.280 & 0.002 & 0.143 & \\
\hline OR, odds ratio;Cl, confidence interval; DS, Durie-Salmon; ISS, International Staging System \\
\hline
\end{tabular}

\section{Discussion}

At present, the relationship between the existence and number of circulating tumor cells in peripheral blood of malignant tumor and tumor genesis, efficacy, metastasis and prognosis has become a hotspot of tumor biology research. Studies have suggested that CMCs in peripheral blood of MM patients can be involved in disease metastasis and recurrence, thus affecting prognosis and treatment [17]. Circulating tumor plasma cells can be detected in patients with symptomatic MM [3, 7], smoking MM [18], monoclonal immunoglobulin disease (MGUS) [19] and primary amyloidosis [20]. However, the number of CMCs in symptomatic MM is relatively high [5], but significantly less than that in MMCs. It is difficult to accurately measure the number of CMCs by general detection methods. More than $90 \%$ of MM patients have abnormal plasma cell phenotype [21]. In this study, according to the immunophenotypic characteristics of MM patients, FCM was used to detect CMCs, and the number of cells obtained increased to 1 million, with a sensitivity of $1.0003 \times 10^{-4}$, which was basically consistent with the report of Gonsalves et al. [7].

Previous studies have shown that using FCM to detect CMCs can reflect tumor load, which is of great significance for MM staging, prognosis and efficacy evaluation. Gonsalves et al. [6, 7] found that CMCs can reflect tumor load and proliferation activity, and the application of CMCs can redefine high-risk MM patients. Nowakowski et al. [22] found that CMCs can independently affect patient survival, which is consistent with ISS staging system. Vagnoni et al. [3] found that CMCs can be used as a poor prognostic factor for patients with primary MM at cytogenetic risk. Dingli et al. [23] used FCM to detect CMCs in MM patients who had received allogeneic hematopoietic stem cell transplantation and found that CMCs was an independent risk factor. Teresa et al. [14] found higher overall levels of cyVEGF in MM patients, which 
may indicate that increased VEGF in MM may be at least partially related to increased VEGF production by these plasma cells and may lead to higher levels of angiogenesis and disease progression.

In this study, MMCs and CMCs were not detected in $23 \mathrm{CR}$ patients, while the positive detection rates of CMCs in the ND/RA group and PR group were $67.9 \%$ and $43.3 \%$, respectively. The number of MMCs and CMCs decreased sequentially in the ND/RA group, PR group and CR group, which indicated that the number of CMCs and MMCs were related to the curative effect. The number of CMCs and MMCs was higher and easier to detect in the ND/RA group, and decreased in PR group, and could not be detected in CR group, which was consistent with the research results of Fujisawa et al. [5]. In this study, there was a positive correlation between the number of CMCs and MMCs in the ND/RA group and PR group. This finding suggests that the number of CMCs is consistent with that of MMCs, and CMCs can reflect tumor cell load. Peceliunas et al. [16] also showed that CMCs can decrease with disease improvement and increase with disease recurrence, so dynamic assessment of the number of CMCs has important clinical value. However, the number of CMCs is much lower than that of MMCs, and the correlation between CMCs and MMCs is not high, which may be related to the characteristics of low proliferation rate of CMCs, low concentration of cytokines promoting proliferation in peripheral blood, absence of bone marrow mesenchymal cells providing nourishment, and poor growth environment [24]. In MM, there is evidence that increased angiogenesis in the bone marrow is an indication of aggressive disease and poor prognosis. Multiple factors including VEGF, interleukin-6, basic fibroblastic growth factor and insulin-like growth-factor-1 appear to be involved in autocrine and paracrine relationships between tumor cells and stroma which influence angiogenesis[25-29]. In this context VEGF is particularly important and has been studied extensively. In addition to its role as a proangiogenic factor, VEGF acts as a regulator of cellular growth, survival, and migration. In the marrow microenvironment VEGF appears to be secreted by the tumor cells as well as by the stromal cells and the secretion by myeloma cells is stimulated by interleukin6 which is, in turn, secreted by stromal cells in response to VEGF stimulation[25] resulting in a positive feedback loop. Adhesion of the myeloma cells to marrow stromal cells results in up-regulation of VEGF as well as interleukin- 6 secretion by the tumor cells and the stromal cells [26]. Myeloma cells in MM secretes VEGF, which acts on vascular endothelial cells and promotes vascular proliferation, and also stimulates stromal cells to produce interleukin-6, a powerful growth factor of myeloma cells, thereby stimulating the increased expression and secretion of VEGF $[25,26]$. This study found that the cyVEGF expression rate of CMCs was significantly higher than that of circulating plasma cells, which indicated that CMCs could produce VEGF increasedly, consistent with the results of Teresa et al. [14].

The clinical characteristics of CMCs-positive MM are controversial. Some studies [22] believe that the number of CMCs is not related to tumor load indicators such as $\beta 2-M G$ and MMCs. On the contrary, some studies $[4,5,7,23]$ suggest that CMCs-positive MM has high levels of $\beta 2-M G$, LDH and MMCs, low Hb level and high DS and ISS stage. In this study, the frequencies of moderate or above anemia, $\beta 2-\mathrm{MG}$ increase, sCrea increase, bone destruction and renal function damage were significantly increased in CMCs positive patients, presenting a high DS and ISS stage, indicating that CMCs is related to high tumor load and stage, which is consistent with Gonsalves et al. [6, 7]. This may be related to the unique cytogenetic abnormality of CMCs [7, 23], the downregulation of the expression of integrins, adhesion 
molecules and activated molecules at rest [30], and the positive correlation between CMCs and the increased number of new microvessels in bone marrow [31], which makes CMCs have a stronger ability to migrate and invade. Multivariate Logistic regression analysis showed that moderate or above anemia and $\beta 2-M G$ increase were independent risk factors for CMCs in MM patients. Multiple studies $[3,4,23]$ have shown that CMCs is a factor of poor prognosis in MM patients. Therefore, it is of great clinical significance to detect CMCs in patients with moderate or above anemia or $\beta 2-\mathrm{MG}$ increase.

Compared with MMCs, detection of CMCs can improve patient compliance. This study still has some limitations. If the sample size was further expanded and the efficacy groups were refined, the number of CMCs in MM patients at different efficacy stages can be obtained.

\section{Declarations}

\section{Statements and Declarations}

The authors have no competing interests to declare that are relevant to the content of this article.

\section{Compliance with ethical standards}

All procedures performed in studies involving human participants were in accordance with the ethical standards of the institutional and/or national research committee and with the 1964 Helsinki Declaration and its later amendments or comparable ethical standards. The study was approved by the Medical Research Ethics Committee of the First Affiliated Hospital of Zhengzhou University ( No. SS-2019-060).

\section{Author contribution}

Baohong Yue conceived the idea and investigated the research study. Ran Tan designed the study, performed research, analyzed and interpreted data, and wrote the manuscript. Yuke Liu and Qiyao Pu guided and validated the statistical analysis. Xueyan Cao helped to analyzed data. Jiwei Li contributed to writing and reviewing of the manuscript.

\section{References}

1. Chinese Hematology A. H. Chinese Society of, A. Chinese Myeloma Committee-Chinese Hematology, The guidelines for the diagnosis and management of multiple myeloma in China(2020 revision). Zhonghua Nei Ke Za Zhi. 2020;59(5):341-6.

2. Wang N, Tesfaluul N, Li J, Gao X, Liu S, Yue B. Enrichment of circulating myeloma cells by immunomagnetic beads combined with flow cytometry for monitoring minimal residual disease and relapse in patients with multiple myeloma. Ann Hematol. 2019;98(12):2769-80.

3. Vagnoni D, Travaglini F, Pezzoni V, e. al., Circulating plasma cells in newly diagnosed symptomatic multiple myeloma as a possible prognostic marker for patients with standard-risk cytogenetics, $\mathrm{Br} \mathrm{J}$ Haematol 170(4) (2015) 523-31. 
4. An G, Qin X, Acharya C, e. al., Multiple myeloma patients with low proportion of circulating plasma cells had similar survival with primary plasma cell leukemia patients, Ann Hematol 94(2) (2015) 257264.

5. Fujisawa M, Suehara Y, Fukumoto K. e. al., Clinical Significance of Circulating Plasma Cells in Myeloma: Multiparameter Flow Cytometric Analysis. Blood. 2015;126(23):5352-2.

6. Gonsalves WI, Morice WG, Rajkumar V, e. al., Quantification of clonal circulating plasma cells in relapsed multiple myeloma, British journal of hematology 167(4) (2014) 500-505.

7. Gonsalves WI, Rajkumar SV, Gupta V, e. al., Quantification of clonal circulating plasma cells in newly diagnosed multiple myeloma: implications for redefining high-risk myeloma, Leukemia 28(10) (2014) 2060-2065.

8. Sanoja-Flores L, Flores-Montero J, Garcés JJ, e. al., Next generation flow for minimally-invasive blood characterization of MGUS and multiple myeloma at diagnosis based on circulating tumor plasma cells (CTPC), Blood Cancer J 8(12) (2018) 117.

9. Li J, Wang N, Tesfaluul N, Gao X, Liu S, Yue B. Prognostic value of circulating plasma cells in patients with multiple myeloma: A meta-analysis. PLoS One. 2017;12(7):e0181447.

10. Kumar SK, Callander NS, Alsina M. e. al., NCCN Guidelines Insights: Multiple Myeloma, Version 3.2018. J Natl Compr Canc Netw. 2018;16(1):11-20.

11. Durie BG, Salmon SE. A clinical staging system for multiple myeloma. Correlation of measured myeloma cell mass with presenting clinical features, response to treatment, and survival. Cancer. 1975;36(3):842-54.

12. Kumar S, Paiva B, Anderson KC. e. al., International Myeloma Working Group consensus criteria for response and minimal residual disease assessment in multiple myeloma. Lancet Oncol. 2016;17(8):e328-46.

13. Rawstron AC, Orfao A, Beksac M, e. al., Report of the European Myeloma Network on multiparametric flow cytometry in multiple myeloma and related disorders, Haematologica 93(3) (2008) 431-438.

14. Kimlinger T, Kline M, Kumar S, e. al., Differential expression of vascular endothelial growth factors and their receptors in multiple myeloma, Haematologica 91(8) (2006) 1033-1040.

15. Chakraborty R, Muchtar E, Kumar SK, e. al., Risk stratification in myeloma by detection of circulating plasma cells prior to autologous stem cell transplantation in the novel agent era, Blood Cancer $\mathrm{J}$ 6(12) (2016) e512.

16. Peceliunas V, Janiulioniene A, Matuzeviciene R, e. al., Circulating plasma cells predict the outcome of relapsed or refractory multiple myeloma, Leuk Lymphoma 53(4) (2012) 641-647.

17. Ghobrial IM. Myeloma as a model for the process of metastasis: implications for therapy. Blood. 2012;120(1):20-30.

18. Bianchi G, Kyle RA, Larson DR, e. al., High levels of peripheral blood circulating plasma cells as a specific risk factor for progression of smoldering multiple myeloma, Leukemia 27(3) (2013) 680-685. 
19. Kumar S, Rajkumar SV, Kyle RA, e. al., Prognostic value of circulating plasma cells in monoclonal gammopathy of undetermined significance, J Clin Oncol 23(24) (2005) 5668-5674.

20. Pardanani A, Witzig TE, Schroeder G, e. al., Circulating peripheral blood plasma cells as a prognostic indicator in patients with primary systemic amyloidosis, Blood 101(3) (2003) 827-830.

21. Paiva B, Vidriales M-B, Cerveró J, e. al., Multiparameter flow cytometric remission is the most relevant prognostic factor for multiple myeloma patients who undergo autologous stem cell transplantation, Blood 112(10) (2008) 4017-4023.

22. Nowakowski GS, Witzig TE, Dingli D, e. al., Circulating plasma cells detected by flow cytometry as a predictor of survival in 302 patients with newly diagnosed multiple myeloma, Blood 106(7) (2005) 2276-2279.

23. Dingli D, Nowakowski GS, Dispenzieri A, e. al., Flow cytometric detection of circulating myeloma cells before transplantation in patients with multiple myeloma: a simple risk stratification system, Blood 107(8) (2006) 3384-3388.

24. Kumar S, Rajkumar SV, Greipp PR, e. al., Cell proliferation of myeloma plasma cells: comparison of the blood and marrow compartments, Am J Hematol 77(1) (2004).

25. Dankbar B, Padró T, Leo R, e. al., Vascular endothelial growth factor and interleukin-6 in paracrine tumor-stromal cell interactions in multiple myeloma, Blood 95(8) (2000) 2630-2636.

26. Gupta D, Treon SP, Shima Y, e. al., Adherence of multiple myeloma cells to bone marrow stromal cells upregulates vascular endothelial growth factor secretion: therapeutic applications, Leukemia 15(12) (2001) 1950-1961.

27. Vacca A, Ribatti D, Presta M, e. al., Bone marrow neovascularization, plasma cell angiogenic potential, and matrix metalloproteinase-2 secretion parallel progression of human multiple myeloma, Blood 93(9) (1999) 3064-3073.

28. Vacca A, Ria R, Ribatti D, e. al., A paracrine loop in the vascular endothelial growth factor pathway triggers tumor angiogenesis and growth in multiple myeloma, Haematologica 88(2) (2003) 176-185.

29. Mitsiades CS, Mitsiades NS, McMullan CJ, e. al., Inhibition of the insulin-like growth factor receptor-1 tyrosine kinase activity as a therapeutic strategy for multiple myeloma, other hematologic malignancies, and solid tumors, Cancer Cell 5(3) (2004) 221-230.

30. Paiva B, Paino T, Sayagues J-M, e. al., Detailed characterization of multiple myeloma circulating tumor cells shows unique phenotypic, cytogenetic, functional, and circadian distribution profile, Blood 122(22) (2013) 3591-3598.

31. Kumar S, Witzig TE, Greipp PR, Rajkumar SV. Bone marrow angiogenesis and circulating plasma cells in multiple myeloma. Br J Haematol. 2003;122(2):272-4.

\section{Figures}


a.

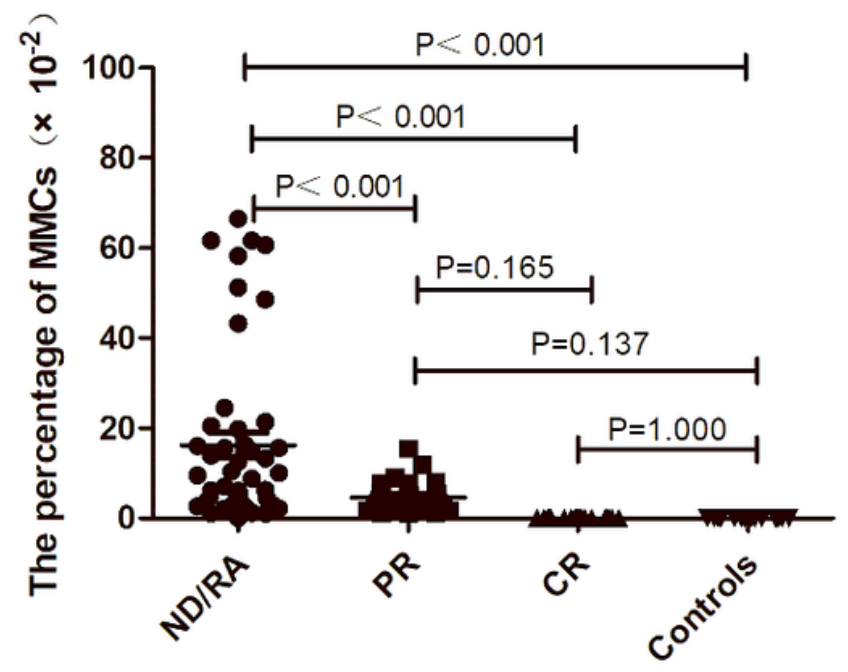

b.

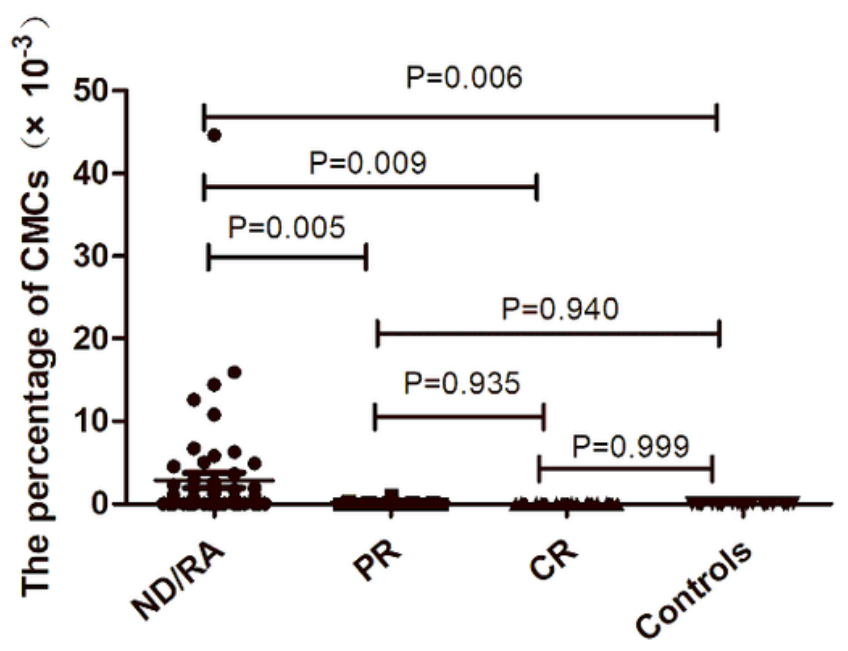

Figure 1

Comparison of myeloma cells ratio among groups. a Comparison of MMCs ratio among groups. $\mathbf{b}$ Comparison of CMCs ratio among groups.

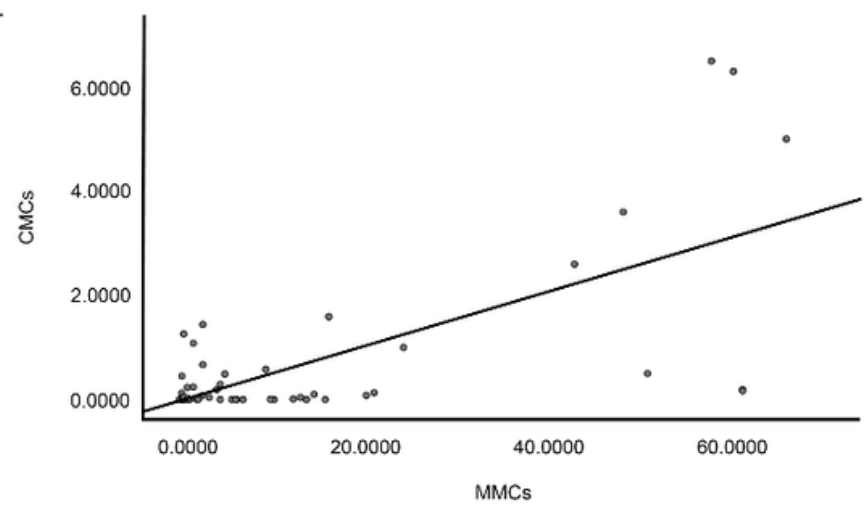

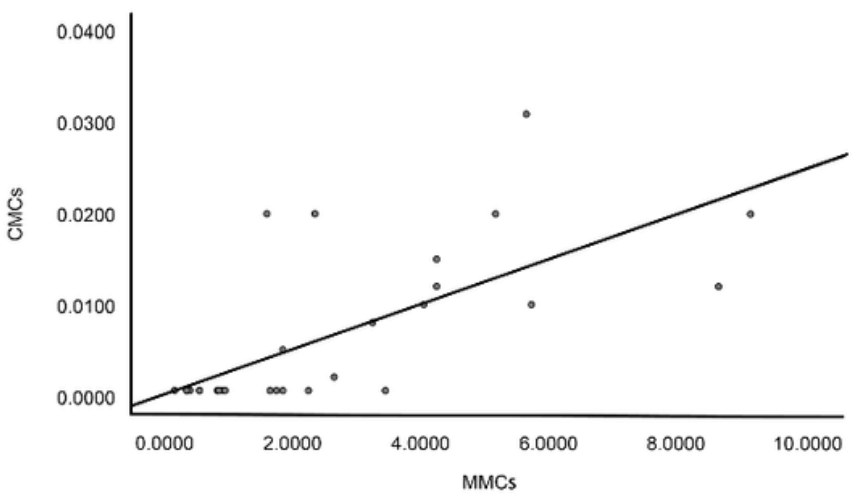

Figure 2

Correlation between CMCs and MMCs ratio. a Correlation between CMCs and MMCs ratio in ND/RA group. $\mathbf{b}$ Correlation between CMCs and MMCs ratio in PR group. 
a.
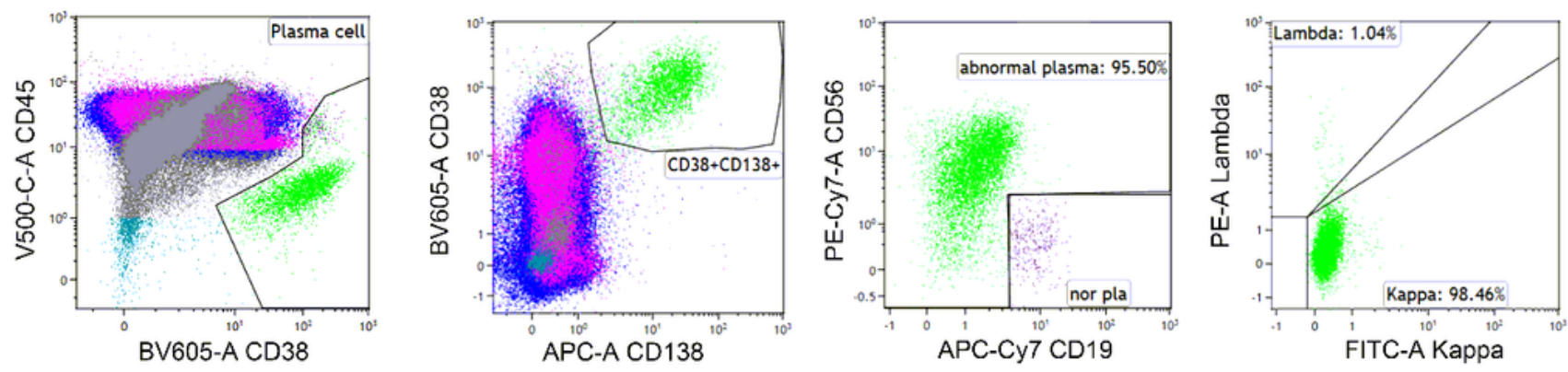

b.
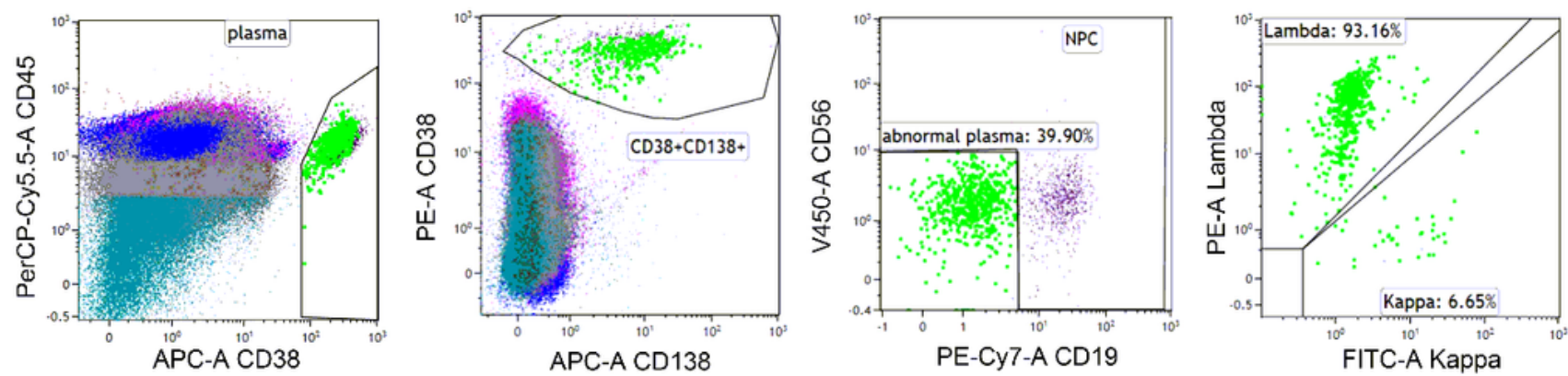

C.
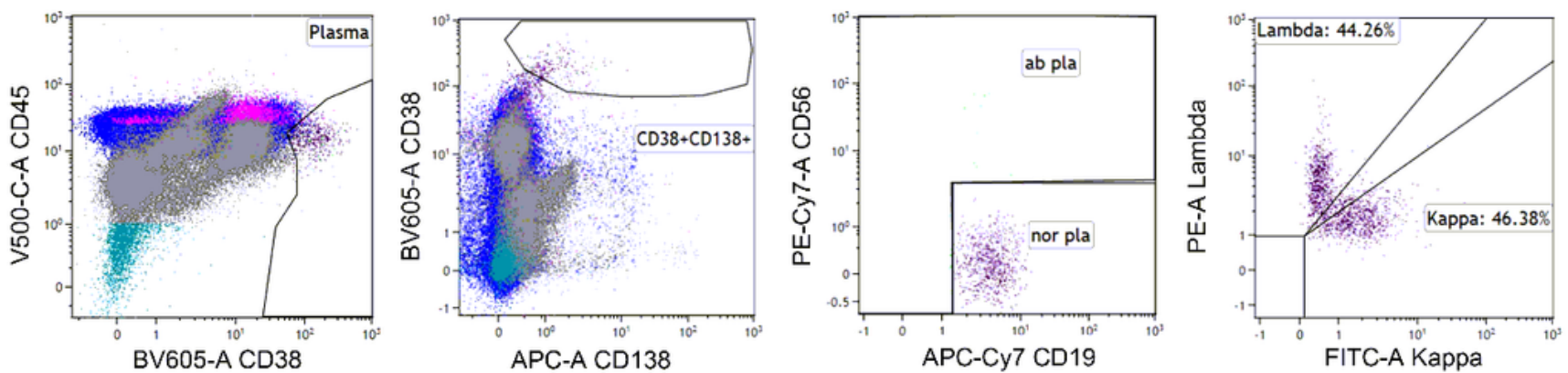

d.
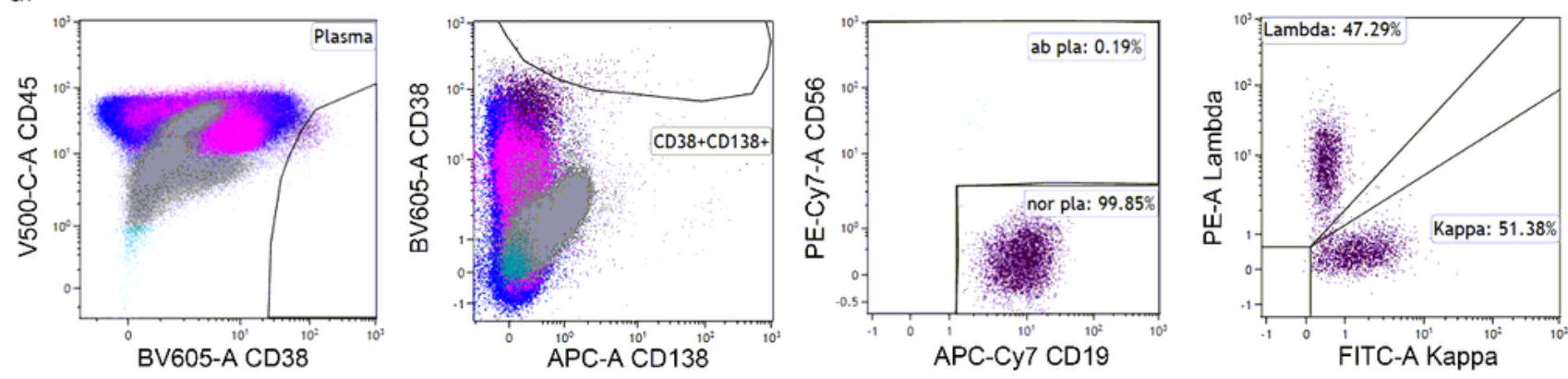

\section{Figure 3}

FCM dual parameter diagram. a FCM dual parameter diagram of MMCs in MM patients. $\mathbf{b}$ FCM dual parameter diagram of CMCs in MM patients. c FCM dual parameter diagram of marrow plasma cells in the control group. $\mathbf{d}$ FCM dual parameter diagram of circulating plasma cells in the control group. 
a.

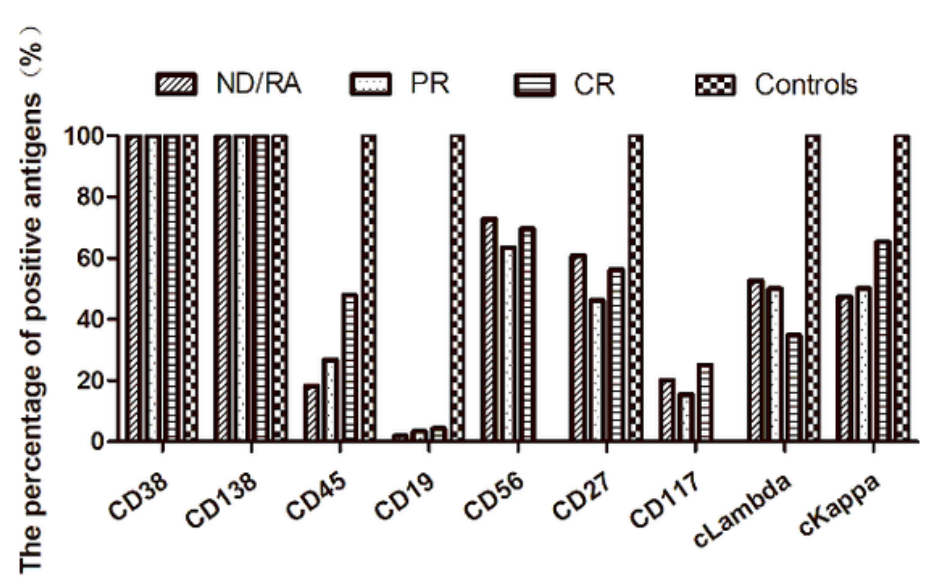

b.

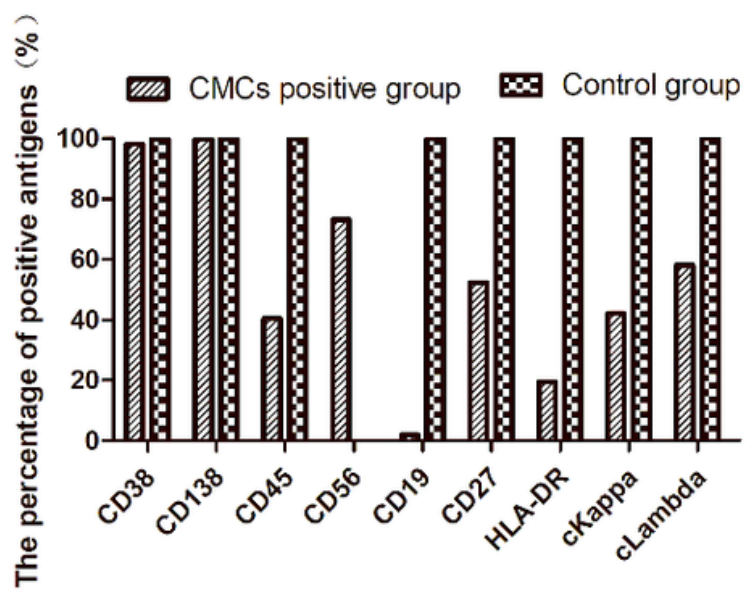

Figure 4

Percentage of positive antigens. $\mathbf{a}$ The percentage of MMCs positive antigens. $\mathbf{b}$ The percentage of CMCs positive antigens.

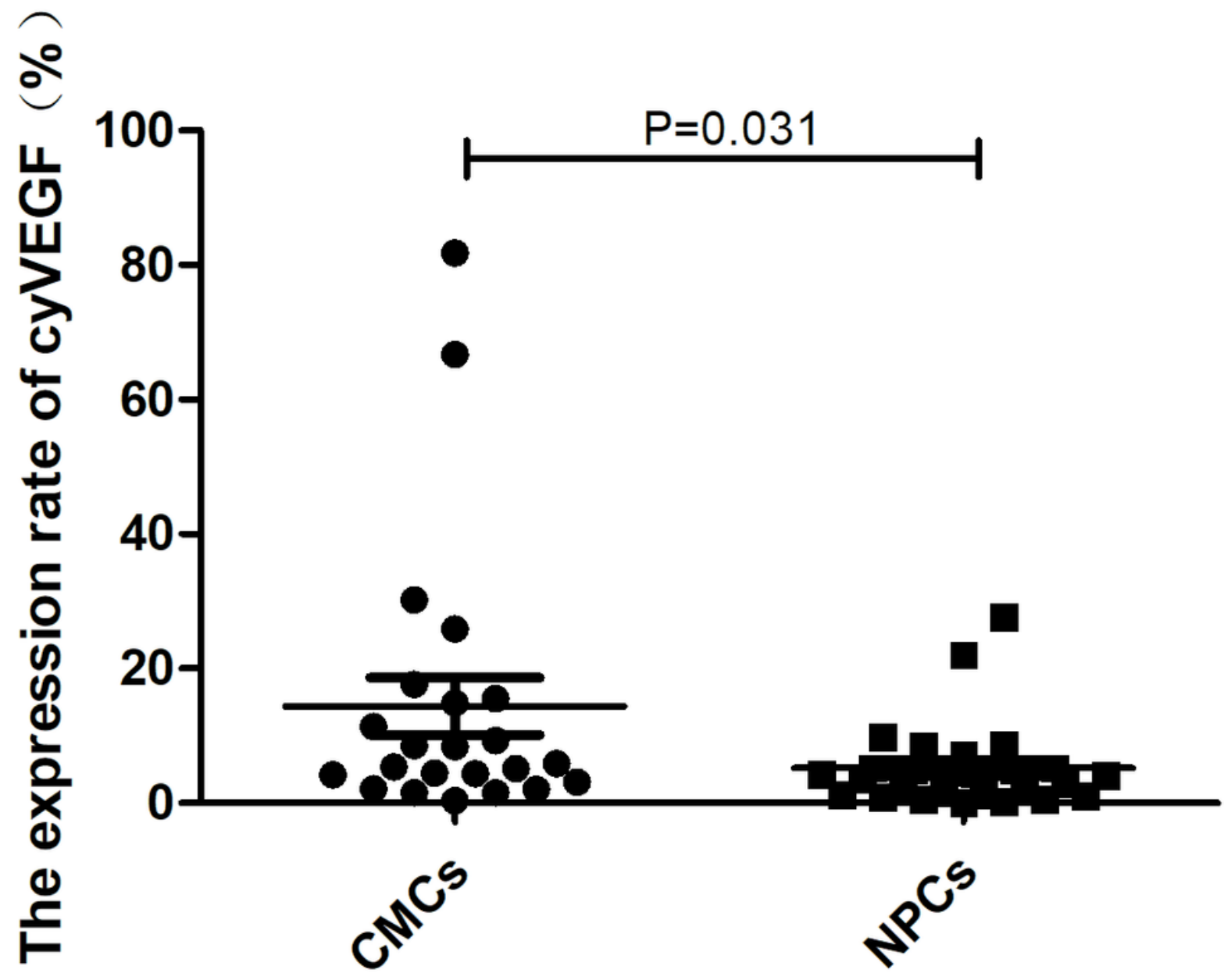


Figure 5

Comparison of cyVEGF expression rate between CMCs and circulating plasma cells. 